\title{
Unfounded worries about cancer in patients attending a routine otolaryngology clinic
}

\author{
Safina Ali MB MRCS B J G Bingham MB FRCS
}

$J$ R Soc Med 2005;98:415

\section{SUMMARY}

To investigate a suspicion that many ear, nose and throat patients have unfounded concerns about cancer, we questioned 50 patients who had attended a routine clinic after screening-out of those with possibly cancer-related features. None of the 50 proved to have cancer. $15(30 \%)$ had been worried about cancer and 7 of these were still worried despite the consultation. Unwarranted fears about cancer are best dealt with by the referring clinician, especially when the wait for an appointment will be long. Such fears also need to be recognized and addressed by the specialist.

\section{INTRODUCTION}

In Scotland as elsewhere in the UK, Government targets require that patients with possible cancer are seen by an appropriate specialist within a narrow time limit. ${ }^{1}$ Consultants in otolaryngology therefore examine outpatient referral letters and give priority to patients whose symptoms are compatible with head or neck cancer. For those not categorized as 'urgent' the wait for a routine appointment can be as long as six months. The present pilot study was prompted by a suspicion that, even in the nonurgent groups, many patients have concerns about cancerperhaps generated or worsened by consultation of internet sources. So they have a long wait before such fears can be allayed by an expert.

\section{METHOD}

A questionnaire was given to 50 consecutive new patients who had been seen at a 'routine' ear, nose and throat clinic (i.e. designated by the consultant as unlikely to have cancer). They were asked whether, before the consultation, they had been concerned that they might have cancer and whether this concern persisted after the consultation. The answers were yes or no. We reviewed all the case notes to determine principal symptoms and eventual outcomes.

Department of Otolaryngology, Southern General Hospital, Glasgow G51 4TK, Scotland, UK

Correspondence to: Miss Safina Ali

E-mail: safinaali@doctors.org.uk
Approval by our local ethics committee is not required for studies of this kind.

\section{RESULTS}

All 50 patients completed the questionnaire - 22 men, 28 women (age range 18-77 years). None proved to have cancer. 15 (30\%) said they had worried that their principal symptom might be due to cancer, and 7 had not lost this fear after the consultation. Fear of cancer was most prevalent in patients with neck symptoms $(5 / 6)$ and least in those with ear symptoms $(1 / 13)$.

\section{DISCUSSION}

On the evidence of this small survey, many patients attending routine ear, nose and throat clinics have a hidden cancer worry. Referring clinicians need to be aware of such anxieties and address them early, so as to prevent needless worry during the wait for an appointment. Moreover, specialists need to be aware of these seemingly unwarranted fears and deal with them. The senior author was greatly troubled by the finding that only half his patients had their cancer concern lifted by the consultation. In consequence, we now ask most patients directly whether they are worried about cancer and reassure them categorically whenever possible.

\section{REFERENCE}

1 Cancer in Scotland: Action for Change [www.scotland.gov.uk] 